\title{
Correction to: Random Self-modifiable Computation
}

\author{
Michael Stephen Fiske
}

\section{Correction to:}

Chapter 27 in: H. R. Arabnia et al. (eds.), Advances in Software Engineering, Education, and e-Learning, Transactions on Computational Science and Computational Intelligence, https://doi.org/10.1007/978-3-030-70873-3_27

The DOI in the reference 12 was published incorrectly and has been corrected to read as mentioned below:

https://doi.org/10.1017/bs1.2019.56 